REVIEW

\title{
Hair as a biomarker for exposure to tobacco smoke
}

\section{W K Al-Delaimy}

Tobacco Control 2002;11:176-182

This article provides an overview of the hair nicotine biomarker for assessment of exposure to tobacco smoke, with emphasis on environmental tobacco smoke (ETS). Measurement of nicotine in hair can be an informative tool for research looking at ETS and related illnesses. There are still unresolved issues in relation to this biomarker such as influence of hair treatment, hair colour, and growth rate on nicotine levels in hair, which need to be addressed in order to further refine this biomarker for exposure assessment. Nevertheless, hair nicotine promises to be a valid and reliable measure of longer term exposure that can be readily applied in epidemiological studies of exposure to tobacco smoke, and more specifically ETS, and its risk to health.

$\mathrm{N}$ icotine is the principal identifying constituent of tobacco, and most studies assessing tobacco smoke exposure (whether active or passive) have looked for methods of measuring nicotine or one of its metabolites in the human body. Levels of nicotine in hair have been suggested by several studies as a possible marker of long term smoke exposure. ${ }^{1-4}$ The relatively long term (up to several months) exposure assessment is the major advantage of this approach, and one that is particularly relevant to epidemiological studies of disease aetiology. This paper reviews this biomarker and its importance as a measure of environmental tobacco smoke (ETS) exposure in the epidemiological setting, examining issues ranging from the physiology of hair to the possible disadvantages of this approach. The definition of ETS in this review is: the tobacco smoke produced by an active smoker (from the exhalation of smoked tobacco; known as mainstream smoke, and that produced by the burning end of the cigarette; known as sidestream smoke) that is inhaled by non-smokers.

The Medline database was searched from 1966 to May 2001 using the keywords "nicotine" and "hair". The overview is divided into four parts: the first describes the physiology of human hair; the second part reviews published literature on the topic (mainly summarised in table 1), the third part describes in detail aspects related to hair as a biomarker of tobacco smoke exposure, and the fourth part outlines the advantages and disadvantages of this biomarker.

Health, Boston, MA 02115, USA;

wael@hsph.harvard.edu

Received 14 March 2001 and in revised form 3 May 2002.

Accepted 5 May 2002

\section{PHYSIOLOGY OF HUMAN HAIR}

Hair physiology is rather complex and not very well understood. However, there seems to be agreement that the mean (SD) rate of hair growth of the scalp is generally $1(0.3) \mathrm{cm}$ per month..$^{5-8}$ Hair growth rate has been calculated as $1.1 \mathrm{~cm} /$ month using drug markers incorporated into the hair through the systemic circulation.' The anatomical location of the hair is the most important factor in hair growth rate. Scalp hair grows more quickly than pubic or axillary hair, and $85-90 \%$ of it is continuously found in the growing stage. The back of the scalp is the area with most uniform growth pattern; it is therefore the recommended site for sample collection. This is because the continuous growth provides updated information on exposure, and the limited percentage of non-growing hair minimises variability of results. ${ }^{10-12}$ Further details on hair growth stages can be found in references 10 and 11 . In general, scalp hair grows faster in women than in men, ${ }^{13}$ which may be related to female hormones. Other factors such as race and age may also affect hair growth rate, but there is no strong evidence in the literature.

Bearing in mind these sources of variability, each $\mathrm{cm}$ of scalp hair reflects approximately one month of past exposure. ${ }^{6}$ The $\mathrm{cm}$-by-cm distribution of nicotine has been found to approximately match the self report of the month-by-month mean number of cigarettes smoked daily (fig l). ${ }^{14}$ Findings from other studies are similar. ${ }^{615}$ Therefore, when using hair samples for analysing "time related exposure" to nicotine or other external substances, it is important to measure the length of the analysed hair segment from the scalp. However, if the aim is to determine the concentration of nicotine per weight of hair as a measure of usual exposure, regardless of the time of exposure, or if history of past exposure was constant, then the length of hair sample becomes irrelevant

\section{STUDIES OF HAIR NICOTINE AS A BIOMARKER OF TOBACCO EXPOSURE}

The first recorded use of hair as a biomarker of exposure was in the mid 19th century ${ }^{16}$ when arsenic was detected in the hair of a deceased person. However, it was Baumgartner et al $^{17}$ in 1979 who laid the foundation for hair analysis in modern times, applied first to testing for opiates with the radioimmunoassay method. Ishiyama et $a l^{18}$ in 1983 were the first to report the presence of nicotine in hair of humans. Since then a number of studies from different countries have investigated the use of hair for measuring exposure to nicotine. Table l summarises these studies.

The level of active smoking can be simply assessed by calculating the self reported number of cigarettes smoked by an individual in a given period. Several studies have compared the self reported number of cigarettes smoked per day with nicotine levels in hair and found significant 


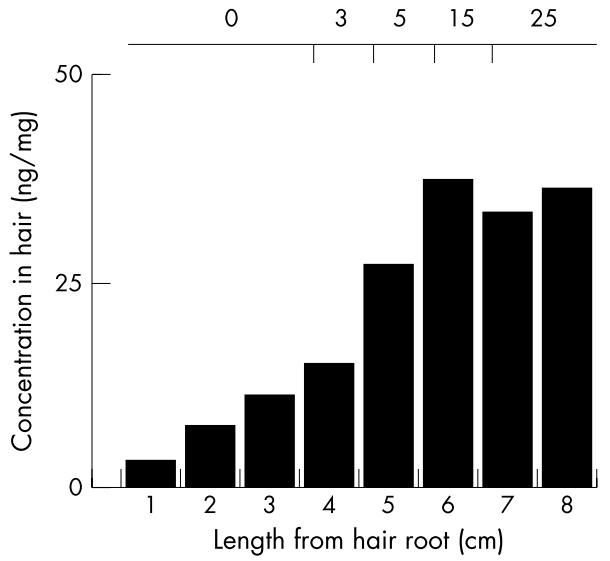

Figure 1 Axial distribution of nicotine along hair shafts collected from a subject who participated in a smoking cessation programme. A scalp hair sample was collected at the end of the programme and cut into $1 \mathrm{~cm}$ lengths successively from the scalp ends. The mean numbers of cigarettes consumed daily reported by the subject every month is shown above the graph. Reprinted from Uematsu ${ }^{14}$ with permission from publisher.

associations. ${ }^{219} 20$ Mizuno et al ${ }^{19}$ compared cigarette smoking history with hair nicotine levels and found it was "imprinted" along the hair shaft, while Uematsu described the hair as a "tape recorder" that continues to store data of the individual exposure to nicotine. ${ }^{14}$ To test the reliability of the hair nicotine method, Zahlsen and Nilsen ${ }^{21}$ carried out a one year follow up of 20 adult subjects in Norway and showed reproducibility of hair nicotine measurements over time. Hair samples were collected twice, 12 months apart, from the same subjects and indicated that the method is sufficiently sensitive to detect changes in active and ETS exposure levels. This finding was also supported by others. ${ }^{22}$

However, other studies have not found an association between active smoking and hair nicotine levels. ${ }^{23}$ In a group of mothers and their newborns in Toronto, Canada, no relation was found between the number of cigarettes smoked by the mothers and their hair nicotine levels, or that of their corresponding neonates. ${ }^{25}$ These studies were concerned with intrauterine exposure to maternal tobacco smoke exposure (whether active or passive). However, they found a strong correlation between maternal and neonatal hair nicotine levels $(r=0.78, \mathrm{p}<0.01)$. Neonates from non-smoking mothers had significantly lower hair nicotine levels than neonates of smoking mothers $(p<0.001)$. Similar findings were reported in another study by the same group (fig 2$)^{23}$ and in other studies (table 1).

The first study to compare urine cotinine levels to hair nicotine levels ${ }^{1}$ looked at hair nicotine and urine cotinine as biomarkers of ETS in a population of 94 children aged 1-3 years from Norway. They reported that nicotine in children's hair correlated more closely with parental smoking history $(r=0.64, \mathrm{p}<0.0001)$ than did cotinine in urine $(r=0.50$, $\mathrm{p}<0.0001)$. Smoking and non-smoking parents could be differentiated through analysis of the nicotine levels in their children's hair. This differentiation was not possible using urine cotinine levels. The median hair nicotine level of children exposed to more then 10 cigarettes per day was 12.4 times higher than levels in those not exposed, while the median of those exposed to less than 10 cigarettes was 3.4 times higher than the unexposed. ${ }^{1}$ The advantages of hair nicotine over the more widely used urine cotinine method was also found in our recent study, ${ }^{26}$ involving the largest sample published to date.

Recently, Nafstad et al ${ }^{27}$ applied hair nicotine levels as a biomarker to predict the effect of ETS exposure from the mother during pregnancy on the size of the newborns. They were able to demonstrate an increased risk of small-forgestational age at birth among exposed children (odds ratio (OR) of upper quartiles with higher nicotine levels compared to the reference lower quartile was $4.2,95 \%$ confidence interval (CI) 1.5 to 11.5 ). We recently applied hair nicotine as a biomarker of exposure of bar and restaurant staff to ETS from their work place. ${ }^{28}$ We found a significant difference in hair nicotine levels of non-smokers according to their smoking policy at work $(p<0.001)$, with non-exposed non-smokers having minimum levels in their hair and heavily exposed non-smokers having levels as high as active smokers (table 1). Such findings would not have been possible using questionnaire measures of exposure alone. The limitation of questionnaires to predict exposure in relation to the place of smoking at a group of children's households was demonstrated in another study, where we found that parents who reported smoking outside the house and never near their children were exposing their children to tobacco smoke at much higher levels than those who never smoked. ${ }^{29}$

The results from these studies show promise of a reliable and valid new method of ETS exposure measurement. The exposure-dose relation with reported history of exposure indicate appropriateness of the use of hair nicotine for collecting objective results from studies investigating the health effects of ETS. Questionnaires still have advantages of low cost and availability for researchers and should be considered complementary to the hair nicotine measures in epidemiological studies that use this biomarker.

\section{HAIR AS A BIOMARKER OF NICOTINE EXPOSURE Metabolism of nicotine}

Cigarette smoke constituents (including nicotine) enter the body by inhalation and are then absorbed into the systemic circulation. Nicotine (a tertiary amine composed of a pyridine and pyrolidine ring) is lipid soluble ${ }^{30}$ and therefore has a large distribution volume in the body $(2-3$ litres $/ \mathrm{kg})$ and readily permeates cell membranes. Almost $80 \%$ of nicotine is metabolised in the liver by cytochrome 4450 enzyme to cotinine. ${ }^{3132}$

It has been suggested that there is a difference in the metabolism of nicotine between smokers and non-smokers; conversion of nicotine to cotinine and its elimination in urine can be more rapid among smokers than non-smokers. ${ }^{33}$ However, based on laboratory studies measuring body clearance rates of labelled nicotine and cotinine among smokers and non-smokers, it has been argued that the pharmacokinetics of nicotine and cotinine are similar in the two groups. ${ }^{34}$ Other factors such as race or ethnicity may contribute to differences in body metabolism of nicotine to cotinine and uptake by hair. In Toronto, 48 "Western and Eastern Indian" children had higher levels of cotinine in their hair than 112 European children with similar reports of exposures to ETS. ${ }^{35}$ The colour of hair was not related to a difference in cotinine levels. The authors attributed this racial difference in cotinine hair levels to slower metabolism of nicotine by Indian children, leading to a longer duration of circulating nicotine and cotinine serum levels which contributed to higher hair levels of these compounds. Three papers ${ }^{36-38}$ have reported similar findings for active smoking among African American adults compared with Americans of European origin. Therefore, ethnicity or race may be an important contributor in nicotine metabolism variability, as may the type and texture of hair in relation to race be related to difference in uptake of nicotine, an area which needs further investigation.

Biological variability in metabolism normally occurs for all xenobiotic agents. Nicotine inhaled into the body follows a biphasic pattern beginning with a short distribution phase (5-10 minutes) and a longer elimination phase with high interindividual variation (70-140 minutes). ${ }^{39}$ The nicotine is incorporated into hair as long as it is present in the circulation; therefore, nicotine collected in hair is representative of the 
Table 1 Main studies published on hair nicotine and cotinine levels in relation to reported exposure

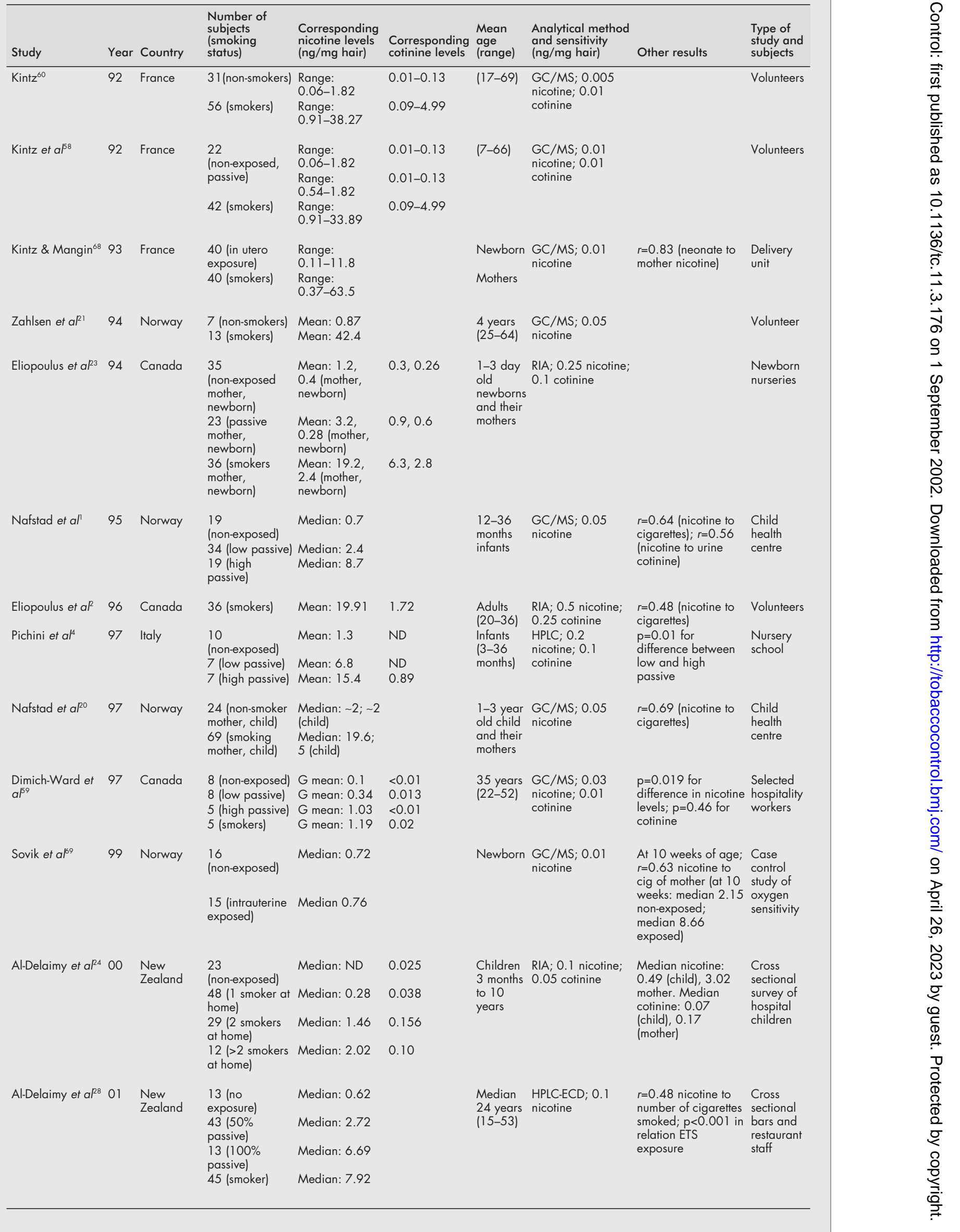


Table 1 Continued Main studies published on hair nicotine and cotinine levels in relation to reported exposure

\begin{tabular}{|c|c|c|c|c|c|c|c|c|c|}
\hline Study & Year & Country & $\begin{array}{l}\text { Number of } \\
\text { subjects } \\
\text { (smoking } \\
\text { status) }\end{array}$ & $\begin{array}{l}\text { Corresponding } \\
\text { nicotine levels } \\
\text { (ng/mg hair) }\end{array}$ & $\begin{array}{l}\text { Corresponding } \\
\text { cotinine levels }\end{array}$ & $\begin{array}{l}\text { Mean } \\
\text { age } \\
\text { (range) }\end{array}$ & $\begin{array}{l}\text { Analytical method } \\
\text { and sensitivity } \\
\text { (ng/mg hair) }\end{array}$ & Other results & $\begin{array}{l}\text { Type of } \\
\text { study and } \\
\text { subjects }\end{array}$ \\
\hline Al-Delaimy et $a^{26}$ & 01 & $\begin{array}{l}\text { New } \\
\text { Zealand }\end{array}$ & $\begin{array}{l}101 \text { (no } \\
\text { exposure) } \\
69 \text { (smoking } \\
\text { out) } \\
127 \text { (smoking } \\
\text { in) }\end{array}$ & $\begin{array}{l}G \text { mean: } 0.58 \\
G \text { mean: } 2.63 \\
G \text { mean: } 5.62\end{array}$ & & $\begin{array}{l}3-27 \\
\text { months }\end{array}$ & $\begin{array}{l}\text { HPLC-ECD; } 0.1 \\
\text { nicotine }\end{array}$ & $\begin{array}{l}r=0.7 \text { nicotine to } \\
\text { number of cigarettes } \\
\text { smoked }\end{array}$ & $\begin{array}{l}\text { Cross } \\
\text { sectional } \\
\text { hospital } \\
\text { children }\end{array}$ \\
\hline
\end{tabular}

GC/MS, gas chromatography with mass spectrophotometry; G mean, geometric mean; HPLC-ECD, high performance liquid chromatography with electro chemical detection; ND, no detection; RIA, radioimmunoassay.

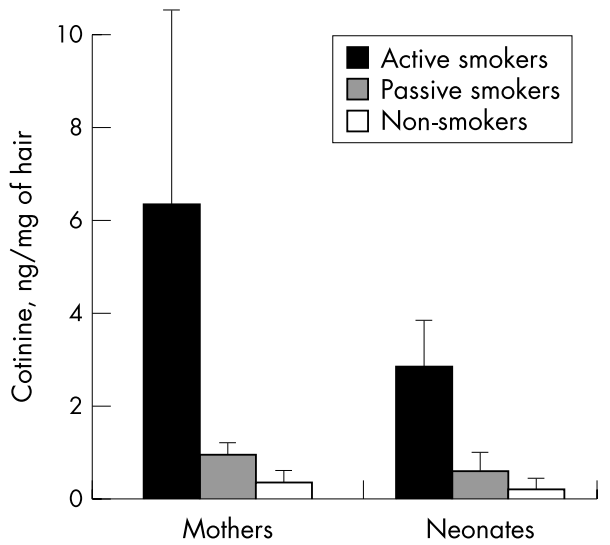

Figure 2 Maternal and their respective neonates' hair concentrations of cotinine according to the maternal smoking habit (active, passive, and non-smokers (that is, non-exposed). Reproduced from Eliopoulos et $a^{23}$ with permission from publisher.

cumulative dose collected gradually over the period of exposure. The fact that $70-80 \%$ of nicotine is cleared by being converted to cotinine ${ }^{39}$ suggests that, over time, the metabolism rate of nicotine may affect levels of nicotine accumulation in hair. This is not expected to cause wide interindividual variability because of the slow growth rate of hair, a concept indirectly supported by findings from Eliopoulos et $a l^{2}$ who reported that levels of nicotine in hair were not correlated to (the more variable) serum nicotine levels of 36 adult smokers $(r=0.24, \mathrm{p}=0.19)$ at a single point of time. The poor correlation between hair nicotine and serum nicotine levels can be expected because the serum levels depend on the instant the blood sample was drawn in relation to the most recent exposure ${ }^{40}$ - whether it was during the peak serum level or after nicotine was metabolised and levels had tapered downwhile nicotine levels in hair are not related to such irregularities because of the slow hair growth rate. (The concentration of nicotine in hair is calculated by dividing the amount of nicotine detected by the weight of the hair sample. ${ }^{21}$ )

When there is interest in time related exposure, hair can be cut in relation to time (so the proximal $1 \mathrm{~cm}$ of hair to the scalp would represent last month's exposure); it will give the average dose of exposure, which is defined as the cumulative exposure divided by the duration of exposure. ${ }^{41}$ The average nicotine dose of hair is therefore less vulnerable to everyday variability of exposure, metabolism, or elimination of nicotine than other biomarkers. For example, measure of dose in urine cotinine represents the average dose for the previous 1-3 days, which makes this measure more prone to the influence of irregularities in ETS exposure and interindividual variability. ${ }^{42-44}$

\section{Hair uptake of nicotine and other substances}

The mode of uptake of drugs by hair has been strongly debated in the literature. ${ }^{45}{ }^{46}$ Most argue that hair mainly takes up external substances like nicotine or its metabolites from the systemic circulation through the hair bulb blood supply. ${ }^{2} 835$ 47-52 It is believed that nicotine moves by passive diffusion from the bloodstream into the growing hair cells at the base of the follicle and then becomes tightly bound in the interior of the hair shaft during subsequent keratogenesis. Therefore, nicotine incorporation in hair is dependent on the average concentration in blood over time, which in turn depends on the dose inhaled or ingested. Knowing the rate at which hair grows, and the distance of the analysed hair section from the scalp, levels in hair may then be related to exposure levels (whether active or passive) in a time dependent manner. ${ }^{14}$

In support of this theory, a constant nicotine concentration has been found along the hair shaft for smokers $(n=36)$ and a decreasing concentration towards the proximal end of the hair shaft for individuals who had quit smoking in recent months $(n=14){ }^{19}$ Similar findings are explained by the uptake of nicotine being related to the inhalation of nicotine, where active smokers who quit or decreased smoking had decreasing hair nicotine levels in their newly grown hair. ${ }^{15}$ Uematsu ${ }^{14}$ demonstrated this same phenomenon (fig 1), and it has also been shown in studies of drugs injected systematically to assess hair growth rates. ${ }^{9}$ Animal studies where subcutaneously injected nicotine was related to its levels in the animal's growing hair also supported this systematic uptake of nicotine..$^{53}$

Others argue that nicotine (and other drugs) is mainly adsorbed into the hair shaft from the ambient environment. ${ }^{21} 4554$ Nilsen et al found similar patterns of increasing nicotine levels at the distal end of hair among five smokers and five non-smokers not involved in smoking cessation programmes. ${ }^{54}$ They attributed these patterns to the adsorption of more nicotine from the environment by the distal end of the hair than the proximal end because of its longer exposure to ETS. The results from Nilsen and colleagues supporting environmental hair adsorption in different studies were mainly based on experimental conditions involving cut hair samples or laboratory animals exposed to varying degrees of nicotine containing vapour or fluid. ${ }^{21}{ }^{54-56}$ The cut hair in these studies was subject to abnormally high levels of nicotine that do not represent the usual indoor levels of exposure. In addition, the cut end of the hair may very well permit the passage of nicotine from the fluid or the vapour into the inside of the hair shaft. In their findings, Nilsen et al found that the rate of environmental uptake by hair decreased with the increasing concentration of nicotine. ${ }^{54}$ It was also reported that short term uptake ( 72 hours) of nicotine in hair is not linear with exposure, while a linear uptake of nicotine seems to exist in the case of long term (eight weeks) exposure to nicotine at low concentration $\left(1.5 \mu \mathrm{g} / \mathrm{m}^{3}\right)$ from controlled air chambers. ${ }^{54}$ 
If adsorption occurs directly from the ambient environment, it is clearly not the only route of entry of nicotine into hair. Gerstenberg et $a l^{53}$ found that $71-90 \%$ of the nicotine from hair samples of laboratory animals that were directly exposed to tobacco smoke was removed by washing,$^{53}$ while Haley and Hoffman found up to 28-fold higher nicotine levels in non-washed hair samples compared to washed hair samples from the same individuals indicating limited, if any, adsorption from external contamination. ${ }^{49}$ These findings have also been reported for drugs such as morphine and cocaine. ${ }^{57}$ Hence, in the laboratory, all hair samples are washed by cleansing solutions before being digested and analysed to avoid contamination from nicotine externally attached to the hair shaft.

It may be that hair does adsorb nicotine directly from the environment after a lengthy contact period, but this route is probably secondary to the main route of systemic circulation nourishing the hair because of the above mentioned observations in human and animal hair studies. Judging from hair washing studies, it seems external adsorption happens for a small fraction from the total external exposure-that is, high sudden external exposure has a limited effect on variability of nicotine levels incorporated into hair shaft if hair samples are washed before analyses. In a recently published study ${ }^{24}$ we found hair nicotine levels to be correlated with hair cotinine levels. Since cotinine is only produced endogenously, this close correlation supports systemic absorption of nicotine into hair. More experimental studies will be required to reach consensus on the percentage of environmental adsorption of nicotine and other chemicals into the hair shaft.

This debate does not directly affect the use of hair nicotine as a biomarker of ETS exposure. Regardless of whether the uptake is systemic or external, nicotine seems to be consistently incorporated in the hair shaft and is available for analysis, and has been found to be well correlated with reports of exposure (table 1). The hair and the face are in close proximity to each other and are therefore expected to be exposed to similar amounts of ETS.

\section{Cotinine in hair}

Cotinine is present in hair, but in much lower concentrations than nicotine ${ }^{4} 45859$ (table 1). In the laboratory, Gerstenberg et $a l^{53}$ reported that nicotine in hair was up to 10 times higher than cotinine even though cotinine levels in plasma were higher than nicotine. Kintz ${ }^{60}$ and others similarly reported the lower cotinine level in hair and its inability to differentiate active and passive exposure groups. However, Eliopoulos et $\mathrm{al}^{2}$ found cotinine in hair to be better correlated to history of cigarette smoking among 36 adults volunteers than nicotine in spite of its being present in much lower concentrations.

As cotinine levels in hair are much lower than nicotine levels, this may reduce detection of ETS exposure, thus explaining the relatively poor correlation to history of exposure among non-smokers in several studies. ${ }^{84} 49505360$ It seems that cotinine is not distributed through the body in exactly the same manner as nicotine. For example, nicotine levels are higher in the breast milk of smoking mothers than in serum, but cotinine levels in breast milk tend to be lower than in serum. ${ }^{61}$ Luck and Nau attributed this finding to a longer half life of nicotine in milk than in serum, and to differences in $\mathrm{pH}$ of the two compounds, and consequently different rates of uptake, $^{61}$ which could similarly explain the differences between nicotine and cotinine incorporation rate into hair.

\section{Hair colour and nicotine uptake}

Hair colour may influence nicotine uptake. Several investigators $\mathrm{s}^{615} 1953$ have reported that white or fair hair has lower nicotine levels than black hair for a similar exposure level. Several participants with grey hair provided black and grey hair simultaneously, and showed lower levels in their grey hair. ${ }^{6}{ }^{15}$ This difference was explained by nicotine having a higher affinity to melanin, which is produced by melanocytes at the hair bulb and incorporated into the cortex of the hair. (This higher affinity to melanin suggests that most of the nicotine in hair is incorporated through systemic circulation by passing through the hair bulb and attaching to the melanocyte granules, which are only present in the cortex of the hair shaft). Gerstenberg et $a l^{53}$ recognised that nonpigmented rat hair had concentrations of nicotine 20 times lower than pigmented rat hair when taken through the systemic route. Hair pigmentation was also related to the levels of nicotine absorbed by cut rat hair samples directly from the external environment, but with a much lower ratio of 1.5:1 (pigmented to non-pigmented). ${ }^{53}$

Others have argued that there is no difference in nicotine (and cotinine) uptake in relation to hair colour. Zahlsen et a ${ }^{55}$ found that nicotine uptake from exposure to different concentrations of nicotine did not differ in relation to the colour of hair. They quantitatively measured melanin levels in hair in order to assess this relation. They also found that the person's age, hair thickness, ${ }^{54}$ and $\operatorname{sex}^{5}$ did not affect nicotine uptake rate. However, their exposure was not systemic but involved cut hair samples. Knight et $a l^{35}$ reported that among 112 white children in Toronto, dark and fair hair had similar cotinine levels for similar reports of exposure, but their findings on cotinine levels do not necessarily apply to nicotine. Further studies are needed to investigate the relation between melanin in hair and nicotine uptake. In the meantime, including hair colour as a covariate in the analyses of hair nicotine results may minimise inter-individual variability.

Cosmetics and other treatments and their effect on hair The ability of the hair to preserve substances incorporated into its shaft may be compromised by externally damaging factors. Several researchers have suggested that the chemical treatment of hair, such as dyeing and permanent waving, can damage the structure of the hair and affect the accurate detection of drugs in it. ${ }^{62}{ }^{63}$ Pichini et al used hair dye, permanent wave, and 30\% hydrogen peroxide and concluded that these chemicals lower nicotine and cotinine levels in hair but do not remove them altogether. ${ }^{64}$ Jurado et al used bleaching and hair dying on eight hair samples of smokers and found that the cuticle of the hair was not damaged after bleaching or dying and nicotine levels were reduced by $30 \% .{ }^{65}$

On the other hand, normal (non-dandruff) shampoo washing does not seem to affect the levels of nicotine in the shaft of the hair. Li and Cheng ${ }^{66}$ found that washing hair samples with shampoo for up to 20 minutes did not affect the internal content of hair. We recently found that adjusting for hair dying history in a multiple regression model did not significantly alter the estimated levels of nicotine in the hair of non-smoking bar and restaurant staff. ${ }^{28}$

Therefore, it is expected that significant cosmetic treatment of the hair (for example, bleaching) will affect hair contents of nicotine, and these variables have to be taken into account when collecting information from adults donating their hair samples for research purposes. Normal hair washing seems to wash away externally attached nicotine rather than the nicotine that is measured by analysis (nicotine incorporated into the shaft of the hair).

\section{ADVANTAGES AND DISADVANTAGES OF HAIR AS A BIOMARKER OF ETS Advantages}

- Hair nicotine provides better information on long term ETS exposure than biomarker measures in urine, saliva, or serum because of the shorter half life of the latter biomarkers. The long term (up to several months) ETS exposure is the usual exposure of interest in health-related studies. 
- The absence of drug metabolism in hair and its fairly uniform growth rate for a given location in the body may provide an historical account of ETS exposure, provided that the concentrations measured in a given hair segment are related to their distance from the scalp (usually the proximal $1-2 \mathrm{~cm}) .{ }^{12124}$

- The hair nicotine biomarker can be analysed with standard laboratory methods, and some of the recently developed methods are cost effective for large epidemiological studies. ${ }^{67}$

- An advantage of nicotine in hair over cotinine in urine is its increased ability to differentiate exposure status because of less variability. ${ }^{26}$

- Hair is easily collected without the need for prior notice to the participants (as is the case when collecting urine samples). No trauma is caused to the donor when collecting the sample (as in collecting blood samples). Samples can be stored readily and inexpensively without deterioration. The nicotine levels in hair are not lowered by storage of samples for a period of up to five years. ${ }^{21}$

- The use of a biomarker such as hair nicotine avoids problems such as recall bias, under reporting or lack of awareness of exposure that may be encountered with questionnaires. In addition, no information is needed on time activity patterns, room air exchange rate, or distance from smokers.

- Nicotine is almost entirely specific to the exposure of interest (ETS) and therefore has better validity than environmental monitors that are used for measuring non-specific pollutants such as suspended particle levels as an indicator of ETS.

- Nicotine in hair can be used for assessment of intrauterine exposure to nicotine by collecting hair samples (if available) from neonates and determining the intrauterine exposure in the later months of pregnancy when fetal hair develops. ${ }^{25} 68$

\section{Disadvantages}

- As with other biomarkers, there are issues of interindividual variation in uptake of nicotine into the hair, and hair nicotine levels may be affected by factors such as irregular hair growth and the hair type and colour. These issues need further research.

- Chemical and physical treatments such as strong hair detergents and permanent waves may influence the integrity of the hair shaft's outer cuticle layer and cause leakage of nicotine from hair. ${ }^{64} 65$

- There are analytical difficulties in extracting nicotine from hair in a consistent and reproducible manner in different laboratories, and there is a lack of reference material for evaluating various laboratory methodologies. ${ }^{64}$

- It is still not understood how nicotine levels in hair relate to the biologically effective dose of ETS.

- Use of the hair biomarker is more costly than questionnaires.

- There are restrictions on cutting hair in some cultures.

- Some infants and adults have scarce scalp hair that may not provide sufficient samples for analysis and assessment of ETS exposure.

\section{CONCLUSIONS}

Hair nicotine measures offer a new approach to ETS assessment in relation to health effects. The data published so far on this method are encouraging, but most are obtained from experimental settings rather than large scale epidemiological studies. Hair nicotine provides the advantage of longer term exposure assessment than other available biomarkers. In addition, the time dependent ETS exposure assessment is unique to hair nicotine. Uncertainties over the mechanism by which drugs are incorporated into hair may have led to caution in the use of hair in forensic toxicology and medicine, but this may not be a serious issue for ETS studies. However, other issues in relation to this biomarker are still unresolved, such as how the hair colour, chemical treatments of hair, or type of hair texture can affect the results and their interpretation. Understanding and adjusting for these variables are expected to improve the precision of this measure. While this needs to be an ongoing process, it should not hamper the current use of this biomarker. It is important to extend the published literature with new studies from different parts of the world, using different study designs, bearing in mind the concerns mentioned above, and attempting to adjust for the contribution of these factors to the variability of results.

In particular, what are most needed are studies that apply this measure of exposure to assessment of ETS health effects. This biomarker may have applications in exposure-disease association assessment. It can also be a useful tool in intervention studies to reduce tobacco consumption or exposure from the environment. Hair nicotine measure of ETS exposure can be a valuable tool in research and clinical settings. With the advancement of molecular epidemiology and the need for more precise methods of exposure measurement, the hair nicotine biomarker will likely become a standard measure of ETS exposure.

\section{ACKNOWLEDGEMENTS}

The author acknowledges the editorial comments and suggestions of Alistair Woodward and Julian Crane on early drafts of this paper.

\section{REFERENCES}

1 Nafstad P, Botten G, Hagen JA, et al. Comparison of three methods for estimating environmental tobacco smoke exposure among children aged between 12 and 36 months. Int J Epidemiol 1995;24:88-94.

2 Eliopoulos C, Klein J, Koren G. Validation of self-reported smoking by analysis of hair for nicotine and cotinine. Ther Drug Monit 1996;18:532-6.

3 Nilsen T, Nilsen OG. Accumulation of nicotine in human hair during long-term controlled exposure to a low concentration of nicotine vapour. Pharmacol Toxicol 1997;81:48-52.

4 Pichini S, Altieri I, Pellegrini $M$, et al. The analysis of nicotine in infants' hair for measuring exposure to environmental tobacco smoke. Forensic Sci Int 1997;84:253-8.

5 Zahlsen K., Nilsen OG. Gas chromatographic analysis of nicotine in hair. Environ Technol 1990;11:353-64.

6 Uematsu T, Mizuno A, Nagashima S, et al. The axial distribution of nicotine content along hair shaft as an indicator of changes in smoking behaviour: evaluation in a smoking-cessation programme with or without the aid of nicotine chewing gum. Br J Clin Pharmacol 1995;39:665-9

7 Que Hee SS. Biological monitoring: an introduction. New York: Van Nostrand Reinhold, 1993.

8 Kintz P, Tracqui A, Mangin P. Detection of drugs in human hair for clinical and forensic applications. Int J Legal Med 1992;105: 1-4.

9 Miyazawa N, Uematsu T. Analysis of ofloxacin in hair as a measure of hair growth and as a time marker for hair analysis. Ther Drug Monit $1992 ; 14: 525-8$.

10 Harkey MR. Anatomy and physiology of hair. Forensic Sci Int 1993;63:9-18.

11 Tosi A, Piraccini B. Biology of Nail. In: Freedberg IM, Eisen AZ, Wolff K, et al, ed. Fitzpatrick's dermatology in general medicine, 5th ed. New York: McGraw-Hill, 1999:231-4.

12 Mangin P, Kintz P. Variability of opiates concentration in human hair according to their anatomical origin: head, axillary and pubic regions. Forensic Sci Int 1993;63:77-83.

13 Saitoh M, Uzuka M, Sakamoto M. Rate of hair growth. Oxford: Pergamon, 1967.

14 Uematsu T. Utilization of hair analysis for therapeutic drug monitoring with a special reference to oflaxacin and to nicotine. Forensic Sci Int 1993;63:261-8.

15 Mizuno A, Uematsu T, Ishikawa T, et al. Clinical outcome of smoking-cessation trial of nicotine chewing gum evaluated by analysis of nicotine in hair. Ther Drug Monit 1997;19:407-12.

16 Casper 1857-8 in Sachs H. History of hair analysis. Forensic Sci Int 1997;84:7-16.

17 Baumgartner AM, Jones PF, Baumgartner WA, et al. Radioimmunoassay of hair for determining opiate abuse histories. J Nucl Med 1979;20:748-52 
18 Ishiyama I, Nagai T, Toshida S. Detection of basic drugs (methamphetamine, antidepressants, and nicotine) from human hair. Forensic Sci 1983;28:380-5.

19 Mizuno A, Uematsu T, Oshima A, et al. Analysis of nicotine content of hair for assessing individual cigarette-smoking behavior. Ther Drug Monit 1993;15:99-104.

20 Nafstad P, Jaakkola JJK, Hagen JA, et al. Hair nicotine concentrations in mothers and children in relation to parental smoking. J Expo Anal Environ Epidemiol 1997;7:235-9.

21 Zahlsen K, Nilsen OG. Nicotine in hair of smokers and non-smokers: sampling procedure and gas chromatographic/mass spectrometric analysis. Pharmacol Toxicol 1994;75:143-9.

22 Seiwert M, Merkel G, Krause C, et al. Determination of nicotine and cotinine in urine and hair of the general population of Germany [abstract]. Epidemiology 1995:6(suppl):S53.

23 Eliopoulos C, Klein J, Phan MK, et al. Hair concentrations of nicotine and cotinine in women and their newborn infants. JAMA 1994;271:621-3.

24 Al-Delaimy WK, Crane J, Woodward A. Questionnaire and hair measurement of exposure to tobacco smoke. J Expo Anal Environ Epidemiol 2000; 10:378-84

25 Koren G, Klein J, Forman R, et al. Biological markers of intrauterine exposure to cocaine and cigarette smoking. Dev Pharmacol Ther 1992; 18:228-36

26 Al-Delaimy WK, Crane J, Woodward A. Is the hair nicotine level a more accurate biomarker of environmental tobacco smoke exposure than urine cotinine? J Epidemiol Community Health 2002;56:66-71.

27 Nafstad P, Fugelseth DL, Qvigstad E, et al. Nicotine concentration in the hair of nonsmoking mothers and size of offspring. Am J Public Health 1998:88:120-4.

28 Al-Delaimy WK, Fraser T, Woodward A. Nicotine in hair of bar and restaurant staff. NZ Med J 2001;114:80-3.

29 Al-Delaimy WK, Crane J, Woodward A. Exposure of children to environmental tobacco smoke in the home: effect of avoidance strategies as measured by hair nicotine levels. Arch Environmental Health 2001;56:117-22.

30 Oldendorf WH. Lipid solubility and drug penetration of the blood brain barrier. Proc Soc Exp Biol Med 1974;147:813-16.

31 Benowitz NL, Jacop PI. Metabolism of nicotine to cotinine studies by a dual stable isotope method. Clin Pharmacol Ther 1994:56:483-93.

32 Idle JR. Titrating exposure to tobacco smoke using cotinine - a minefield of misunderstandings. J Clin Epidemiol 1990;43:313-7.

33 Haley NJ, Sepkovic DW, Hoffmann D. Elimination of cotinine from body fluids: disposition in smokers and non-smokers. Am J Public Health 1989;79:1046-8.

34 Benowitz NL, Jacob P. Nicotine and cotinine elimination pharmacokinetics in smokers and nonsmokers. Clin Pharmacol Ther 1993;53:316-23.

35 Knight JM, Eliopoulos C, Klein J, et al. Passive smoking in children. Racial differences in systemic exposure to cotinine by hair and urine analysis. Chest 1996;109:446-50.

36 Wagenknecht LE, Burke GL, Perkins LL, et al. Misclassification of smoking status in the CARDIA study: a comparison of self-report with serum cotinine levels. Am J Public Health 1992;82:33-6.

37 Perez-Stable EJ, Herrera B, Jacob Pl, et al. Nicotine metabolism and intake in black and white smokers. JAMA 1998;280:152-6.

38 Benowitz NL, Perez-Stable EJ, Fong I, et al. Ethnic differences in $\mathrm{N}$-glucuronidation of nicotine and cotinine. J Pharmacol Exp Ther 1999:291:1196-203.

39 Kyerematen GA, Damiano MD, Dvorchik BH, et al. Smoking induced changes in nicotine disposition: application of a new HPLC assay fo nicotine and its metabolites. Clin Pharmacol Ther 1982;32:769-80.

40 Caraballo RS, Giovino GA, Pechacek TF, et al. Factors associated with discrepancies between self-reports on cigarette smoking and measured serum cotinine levels among persons aged 17 years or older. Am J Epidemiol 2001;153:807-14.

41 Armstrong BK, White E, Saracci R. Principles of exposure measuremen in epidemiology. Oxford: Oxford University Press; 1992:11.

42 Emerson JA, Hovell MF, Meltzer SB, et al. The accuracy of environmental tobacco smoke exposure measures among asthmatic children. J Clin Epidemiol 1995;48:1251-9.

43 Peterson EL, Johnson CC, Ownby DR. Use of urinary cotinine and questionnaires in the evaluation of infant exposure to tobacco smoke in epidemiologic studies. J Clin Epidemiol 1997;50:917-23.
44 Margolis PA, Keyes $L$, Greenberg RA, et al. Urinary cotinine and parent history (questionnaire) as indicators of passive smoking and predictors of lower respiratory illness in infants. Pediatr Pulmonol 1997;23:417-23.

45 Kidwell DA, Blank DL. Comments on the paper by WA Baumgartner and VA Hill: sample preperation techniques. Forensic Sci Int 1993:63:137-43.

46 Baumgartner WA, Hill VA. Comments on the paper by DL Blank and DA Kidwell: external contamination of hair by cocaine: an issue in forensic interpretation. Forensic Sci Int 1993;63:157-60.

47 Du Pont RL, Baumgartner WA. Drug testing by urine and hair analysis: complementary features and scientific issues. Forensic Sci Int 1995;70:63-76.

48 Stout PR, Ruth JA. Deposition of ${ }^{\mathrm{H}-3}$ cocaine, ${ }^{\mathrm{H}-3}$ nicotine, and H-3flunitrazepam in mouse hair melanosomes after systemic administration. Drug Metab Dispos 1999:27:731-5.

49 Haley NJ, Hoffmann D. Analysis for nicotine and cotinine in hair to determine cigarette smoker status. Clin Chem 1985;31:1598-600.

50 Kintz P, Kieffer I, Messer J, et al. Nicotine analysis in neonates' hair for measuring gestational exposure to tobacco. J Forensic $\mathrm{Sci}$ 1993:38: 119-23

51 Martinez F, Poet TS, Pillai R, et al. Cocaine metabolite (benzoylecgonine) in hair and urine of drug users. J Anal Toxicol 1993; 17:138-42.

52 Knight JM, Eliopoulos C, Klein J, et al. Pharmacokinetic predisposition to nicotine from environmental tobacco Smoke - a risk factor for pediatric asthma. J Asthma 1998:35: 113-17.

53 Gerstenberg B, Schepers G, Voncken P, et al. Nicotine and cotinine accumulation in pigmented and unpigmented rat hair. Drug Metab Dispos 1995; 23:143-8.

54 Nilsen T, Zahlsen K, Nilsen OG. Uptake of nicotine in hair during controlled environmental air exposure to nicotine vapour: evidence for a major contribution of environmental nicotine to the overall nicotine found in hair from smokers and non-smokers. Pharmacol Toxicol 1994; 75: 136-42.

55 Zahlsen K, Nilsen T, Nilsen OG. Interindividual differences in hair uptake of air nicotine and significance of cigarette counting for estimation of environmental tobacco smoke exposure. Pharmacol Toxicol 1996;79: 183-90.

56 Nilsen T, Nilsen OG. Accumulation of nicotine in human hair during long-term controlled exposure to a low concentration of nicotine vapour. Pharmacol Toxicol 1997;81:48-52.

57 Wang WI, Cone EJ. Testing human hair for drug abuse. IV: environmental cocaine contamination and washing effects. Forensic Sci Int 1995;70:39-51

58 Kintz P, Ludes B, Mangin P. Evaluation of nicotine and cotinine in human hair. J Forensic Sci 1992;37:72-6.

59 Dimich-Ward H, Gee H, Braver M, et al. Analysis of nicotine and cotinine in the hair of hospitality workers exposed to environmental tobacco smoke. J Occup Environ Med 1997;39:946-8.

60 Kintz P. Gas chromatographic analysis of nicotine and cotinine in hair. $J$ Chromatogr 1992;580:347-53.

61 Luck W, Nau H. Nicotine and cotinine concentrations in serum and milk of nursing smokers. Br J Clin Pharmacol 1984;18:9-15

62 Baumgartner W, Berka C. Hair analysis for drugs of abuse. Am Assoc Clin Chemistry 1989:10:7-16.

63 Welch MJ, Sniegoski LT, Allgood CC, et al. Hair analysis for drugs of abuse: evaluation of analytical methods, environmental issues, and development of reference materials. J Anal Toxicol 1993; 17:389-98.

64 Pichini S, Altieri I, Pellegrini M, et al. Hair analysis for nicotine and cotinine: evaluation of extraction procedures, hair treatments, and development of reference material. Forensic Sci Int 1997;84:243-52.

65 Jurado C, Kintz P, Menendez M, et al. Influence of the cosmetic treatment of hair on drug testing. Int J Legal Med 1997;1 10:159-63.

$66 \mathrm{Li} \mathrm{Y,} \mathrm{Cheng} \mathrm{L.} \mathrm{Analysis} \mathrm{of} \mathrm{nicotine} \mathrm{in} \mathrm{hair} \mathrm{as} \mathrm{a} \mathrm{biological} \mathrm{marker} \mathrm{for} \mathrm{its}$ exposure. Proceedings of Indoor Air'93 1993;1:687-92.

67 Mahoney GN, Al-Delaimy WK. The measurement of nicotine in hair by reversed-phase high performance liquid chromatography with electrochemical detection. Journal of Chromatography-Biomedical Applications 2001;753:179-87.

68 Kintz P, Mangin P. Evidence of gestational heroin or nicotine exposure by analysis of fetal hair. Forensic Sci Int 1993;63:99-104.

69 Sovik S, Lossius K, Eriksen M, et al. Development of oxygen sensitivity in infants of smoking and non-smoking mothers. Early Hum Develop 1999;56:217-32. 